\section{Immunmodulation wirkt zweifach}

\author{
Die Wirkungsweise von Tacrolimus bei topischer Anwendung \\ scheint sich nicht auf die Hemmung der T-Helfer Zellen zu \\ beschränken, sondern auch auf die der Antigen präsentierenden \\ Zellen zu erstrecken. Damit scheint diese neue Therapieoption \\ direkt an entscheidenden pathophysiologischen Schaltern der \\ atopischen Dermatitis (AD) anzugreifen.
}

acrolimus wird seit Mitte der 90er
Jahre erfolgreich in der Transplanta-
tionsmedizin gegen Abstoßungsreaktio-
nen eingesetzt. Verkürzt dargestellt bil-
det Tacrolimus mit einem so genannten
„FK Binding Protein“ im Zellinnern
der T-Zellen einen Komplex und unter-
drückt damit einen intrazellulären
Stoffwechselweg, der für die Aktivie-
rung von T-Zellen benötigt wird. Eine
zytotoxische Abwehrreaktion unter-
bleibt, das Organ wird nicht abge-
stoßen. Die Vorgänge allein würden
durchaus ausreichen, um die Wirkung
von Tacrolimus bei der AD zu erklären,
denn T-Zellen sind ja auch in die Pa-
thogenese der AD involviert.

Jüngste Studienergebnisse zeigen, dass Tacrolimus in der Haut einen weiteren Ansatzpunkt hat. Neben den THelferzellen werden möglicherweise auch die Langerhans-Zellen (LZ) und die so genannten inflammatorischen dendritischen epidermalen Zellen (IDEC) gehemmt. Beide Zelltypen gehören zu den Antigen-präsentierenden Zellen. Sie kooperieren in der Haut eng mit den T-Helferzellen. Mehr noch: Auf der Oberfläche dieser Zellen befinden sich hochaffine Rezeptoren für das Immunglobulin E (FceRI), welche vermutlich eine zentrale Rolle bei der atopischen Dermatitis spielen.

Eine Arbeitsgruppe von Andreas Wollenberg an der Ludwig-Maximilians-Universität München untersuchte jetzt zusammen mit Forschern der Universität Bonn bei zehn Patienten mit $\mathrm{AD}$ Hautproben von unbehandelten und mit Tacrolimus behandelten Läsionen. In den unbehandelten Läsionen fanden die Wissenschaftler - erwartungsgemäß - eine starke Expression von FceRI. Die aus den Hautproben isolierten LZ hatten zudem eine starke stimulierende Wirkung auf autologe T-
Zellen. Auch dies entspricht den derzeitigen pathophysiologischen Vorstellungen von der $\mathrm{AD}$.

Bisher hätte man jedoch nicht erwartet, dass Tacrolimus hier Veränderungen auslöst - was jedoch klar der Fall ist. Die Autoren fanden gleich mehrfache Hinweise für eine Aktivität von Tacrolimus auf der Ebene der Antigen präsentierenden Zellen. Nicht nur dass die Expression von FceRI sowohl in LZ als auch in IDEC heruntergefahren war, auch die stimulatorische Aktivität der LZ wurde durch Tacrolimus offenbar gehemmt.
Eistoment inbezogen in die Studie waren 242 Asthmapatienten mit einem positiven Hautpricktest für Hausstaubmilben. Sie erhielten oral innerhalb von 24 Stunden dreimal eine C30-Potenz des Allergens oder Plazebo. Die Nachbeobachtungszeit betrug 16 Wochen. Erhoben wurden alle gängigen klinischen Asthmaparameter sowie Parameter zur subjektiven Krankheitseinschätzung.

Bei den meisten Parametern ergab sich kein Unterschied zwischen Plazebo und der C30-Allergen-Verdünnung. Nicht erklärt werden konnten die in der dritten Woche signifikanten Verschlechterungen in der Einschätzung der Krankheitsschwere $(p=0,047)$ und Stimmung ( $\mathrm{p}=0,013)$ in der "Verum"Gruppe. Die Ergebnisse waren unabhängig davon, ob die Patienten an die
Schließlich konnten die Autoren nachweisen, dass die Zahl der IDEC, die in läsionaler Haut erhöht ist, durch Tacrolimus deutlich gesenkt wird. $\mathrm{Ob}$ diese Zellen aus der Epidermis migrieren oder ob Tacrolimus sie in die Apoptose treibt, ist noch unklar.

\section{Fazit}

Es bleibt noch viel Arbeit bis zur vollständigen Klärung der Wirkung von Tacrolimus auf zellulärer Ebene in den Antigen präsentierenden Zellen und von Fragen wie: Wo genau setzt Tacrolimus an? Bilden sich ähnliche Komplexe mit einem „FK Binding Protein“ in LZ und IDEC? Die offensichtliche Hemmung Antigen präsentierender Zellen ist jedoch eine besonders attraktive Therapieoption. rme

Wollenberg A et al. Topical tacrolimus (FK506) leads to profound phenotypic and functional alterations of epidermal antigen-presenting dendritic cells in atopic dermatitis. J Allergy Clin Immunol 2001; 107: 519-25

\title{
Homöopathie wirkungslos
}

\author{
Alternativmedizinische Methoden wie die Homöopathie erfreuen \\ sich bei Patienten großer Beliebtheit. Eine plazebokontrollierte \\ Doppelblindstudie untersuchte die Wirksamkeit einer \\ „homöopathischen Immuntherapie“ bei Asthmatikern.
}

Effektivität der Homöopathie glaubten oder nicht.

\section{Kommentar}

Die Studie attestiert der einmaligen Allergengabe in homöopathischen Dosen Unwirksamkeit bei Asthmapatienten mit Hausstaubmilben-Sensibilisierung. $\mathrm{Ob}$ sich Anhänger der Homöopathie dadurch umstimmen lassen, bleibt fraglich: Die einmalige Anwendung entspricht nicht den Gepflogenheiten der Homöopathie und die C30-Potenz stellt auch hier eine sehr starke Verdünnung dar.

Lewith GT et al.: Use of ultramolecular potencies of allergen to treat asthmatic people allergic to house dust mite: double blind randomised controlled clinical trial. BMJ 2002; 324: 520-23 